\title{
Epigenetic mutations in CDKN2A in western Swedish families with hereditary malignant melanoma
}

\author{
ANNA ERLANDSON ${ }^{1}$, FRIDA APPELQVIST ${ }^{2}$ and CHARLOTTA ENERBÄCK ${ }^{1,2}$ \\ Departments of ${ }^{1}$ Clinical Genetics, and ${ }^{2}$ Dermatology, Sahlgrenska University Hospital, SE-413 45 Göteborg, Sweden
}

Received November 8, 2007; Accepted December 4, 2007

\begin{abstract}
This study aimed to identify the molecular genetic variations associated with an increased risk of hereditary malignant melanoma (HMM) in the western Swedish population. In 68 families with increased hereditary susceptibility to malignant melanoma, we previously reported a low frequency of alterations in the CDKN2A gene, which is regarded as the major melanoma predisposition gene. Among these alterations, we identified a novel mutation in 3 families (Asp108Tyr). In the present study, we focused on the possible role of heritable epimutations as a cause of the silencing of the CDKN2A gene. We used two different technical approaches to detect changes in $\mathrm{CpG}$ methylation in the promoter region of the CDKN2A gene; methylation-specific PCR (MSP) analysis of bisulfiteconverted DNA and methylation-specific multiplex ligationdependent probe amplification (MS-MLPA). From families who tested negative for germ-line CDKN2A mutations, 64 unrelated patients with hereditary melanoma were included in the study. We showed a consistent lack of hypermethylation in the promoter region of CDKN2A in patients with HMM in our western Swedish population. A putative germ-line methylation of the CDKN2A, if any, is therefore likely to be a rare event in hereditary melanoma. This study demonstrates that there are probably additional and as yet unknown genetic factors present in western Swedish HMM families.
\end{abstract}

\section{Introduction}

Approximately $10 \%$ of all cases of cutaneous melanoma are found in families with an increased hereditary predisposition to develop this disease, also known as hereditary malignant melanoma (HMM). Our research aims to identify the molecular genetic variations associated with an increased risk of HMM in the western Swedish population. A specific mutation spectrum has been found in many hereditary diseases in our region.

Correspondence to: Dr Frida Appelqvist, Department of Dermatology, Sahlgrenska University Hospital, SE-413 45 Göteborg, Sweden

E-mail: frida.appelqvist@vgregion.se

Key words: Swedish families, malignant melanoma, CDKN2A
Moreover, there is a higher incidence of both malignant melanoma and dysplastic nevi (DN) in western Sweden compared to other parts of the country. We previously reported a novel mutation (Asp108Tyr) in the CDKN2A gene in 3 families; it segregated with the disease in these families and has previously been detected as a somatic mutation in other cancers, such as bladder cancer (1). Among our families, the prevalence of CDKN2A mutations was $5.9 \%$. This is a significantly lower incidence than that expected based on previous Swedish data showing mutations in 17 out of 100 families of mostly southern Swedish origin (2). It is therefore likely that additional, as yet unknown, melanoma predisposing genetic factors may be revealed by further study of this western Swedish population.

The purpose of the present study was to exclude epigenetic silencing of the CDKN2A gene as a cause of HMM in our population. Epigenetic modification of DNA by promoter methylation, which inhibits the transcription of downstream genes, has been recognized as a common change in human cancers. Recently, in a family putatively affected by nonpolyposis colorectal cancer, several affected family members were shown to carry a heritable germ-line methylation in the MSH2 gene (3). The existence of transgenerational epigenetic inheritance was further supported by Hitchins et al, who showed the transmission of a hypermethylated MLH1 allele in successive generations (4). The suggestion that epimutations may be inherited prompted us to study whether they could be an explanation for hereditary melanoma in patients who test negative for CDKN2A mutations. To address this question, we used both methylation-specific PCR (MSP) analysis of bisulfite-converted DNA, as well as the methylation-specific multiplex ligation-dependent probe amplification (MS-MLPA) method, to detect hypermethylation in the promoter region of the CDKN2A gene in 64 unrelated patients with hereditary melanoma.

\section{Materials and methods}

A total of 64 families with hereditary melanoma participated in this study. These families undergo regular follow-up at the Departments of Dermatology of Sahlgrenska University Hospital in the cities of Göteborg and Uddevalla, on the West coast of Sweden. The frequency of DN was high in the study group and in the normal population, especially in patients with melanoma, 53/60 (88.3\%) of which also have DN. Of the 107 families, $97(90 \%)$ members had at least two first- or seconddegree relatives with malignant melanoma. 

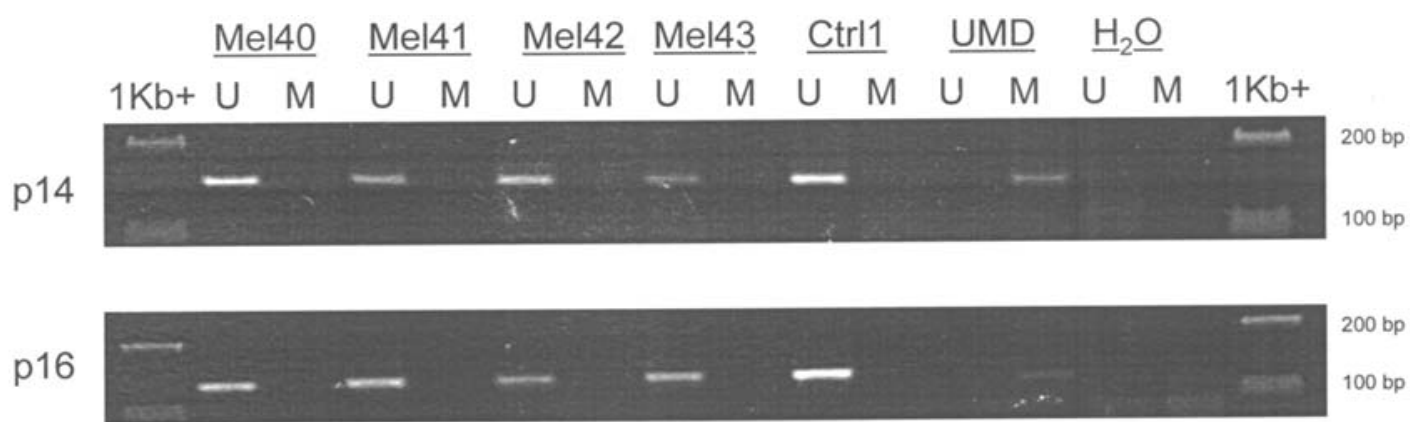

Figure 1. Methylation-specific PCR (MSP) screening for CDKN2A (p14 and p16) promoter methylation in a representative sample of patients with malignant melanoma. Bisulfite-modified genomic DNA from the leucocytes of patients with malignant melanoma and normal controls (healthy blood donors) were used in PCR reactions with the unmethylated- (U) or methylated-specific (M) primer sets. Shown are MSP products from patients 40-43 (Mel40-Mel43) and a healthy blood donor (Ctrl1). Universal methylated DNA (Chemicon International, USA) was used as the positive control in the methylation-specific PCR. The negative control $\left(\mathrm{H}_{2} \mathrm{O}\right)$ was established by replacing DNA with water.

The clinical characteristics of the families have previously been described in more detail (1). In four of the families, DNA sequencing identified a putative germ-line mutation in the CDKN2A gene. Consequently, 64 of the families tested negative for CDKN2A mutations. From each of these 64 families, one patient who had had melanoma was included in the present study. Approval from the ethics committee at Göteborg University was obtained for the study. All patients gave their written informed consent.

DNA was extracted from $300 \mu 1$ of EDTA blood using the Puregene DNA purification system (Gentra Systems, Minneapolis, MN). DNA methylation patterns in the $\mathrm{CpG}$ islands of the CDKN2A gene coding for p14 and p16 were determined by MSP, as described by Herman et al (5). MSP distinguishes unmethylated from methylated alleles in a given gene based on sequence changes produced after bisulfite treatment of DNA, which converts unmethylated (but not methylated) cytosines to uracil, and subsequent PCR using primers designed for either methylated or unmethylated DNA (5). DNA was treated with sodium bisulfite using the EpiTect ${ }^{\circledR}$ Bisulfite Kit (Qiagen, Hilden, Germany). The primer sequences designed for the MSP of the promoter regions of p14 and p16 have previously been described $(6,7)$. CpGenome ${ }^{\mathrm{TM}}$ Universal Methylated DNA, which is enzymatically-methylated human genomic DNA, served as a methylation-positive control (Millipore, Billeria, MA, USA). DNA from healthy blood donors was used as a negative control for methylated genes. PCR products were separated by electrophoresis in $2.0 \%$ agarose gels, stained with ethidium bromide and visualized under UV illumination (Fig. 1).

MS-MLPA is a development of the MLPA method and detects the aberrant methylation of $\mathrm{CpG}$ islands (8). We used MS-MLPA Kits ME001 and ME002 to analyze CpG island methylation in 34 tumor suppressor genes that are frequently silenced by methylation in different tumors (MRC-Holland Amsterdam, The Netherlands). The MS-MLPA probes are similar to ordinary MLPA probes, except that the sequence detected by the MS-MLPA probes contains a recognition sequence for the methylation-sensitive restriction enzyme $H h a \mathrm{I}$. A probe signal will therefore be detected if the site is methylated.

MLPA reactions were performed in thermal cyclers with heated lids, i.e., PTC-100 (Multi) or GeneAmp PCR System 2700 (Applied Biosystems, Foster City, CA). Probemix ME001 and ME002 included 41 probes, 3 of which were located on the CDKN2A and CDKN2B genes, while the remaining 38 detected other tumor suppressors, such as BRCA1 and MLH1. Internal controls for ligation and DNA quantity were included in each reaction from the MLPA Kit.

Sequence pilot version 1.2 software (JSI Medical Systems $\mathrm{GmbH}$, Kippenheim, Germany) was used for quantification analyses, with normalization of the probes not located at the target position on chromosome 9p21.

\section{Results}

With the aim of identifying heritable epimutations in the CDKN2A gene promoter region as a cause of HMM, peripheral blood from 64 unrelated melanoma patients with at least two first- or second-degree relatives with malignant melanoma were analyzed. All the patients lacked germ-line sequence mutations in the CDKN2A gene. To identify epimutations in the CDKN2A promoter region, MSP was performed after bisulfite modification. The region chosen for the MSP spanned the area of greatest CpG density $(6,7)$. The reliability of the method was verified using unmethylated controls (normal PBL from blood donors), as well as a methylated controls (enzymatically methylated human genomic DNA). We found that the methylation levels of the CDKN2A promoter were low, with no significant difference between melanoma patients and controls (Fig. 2).

The conventional MSP used here may have limitations as scoring for methylation based on the visualization of bands can be subjective and is not automated. Therefore, to confirm our findings, we performed MS-MLPA. Instead of the bisulfite modification of DNA to visualize methylation, this method is based on the methylation-sensitive endonuclease digestion of a DNA-probe complex (8). MS-MLPA is a development of the MLPA method and detects changes in CpG methylation of up to 40 sequences in a single reaction (8). It was recently evaluated for Prader-Willi and Angelman syndrome and was shown to produce results concordant with other methylationspecific assays (9).

We used MS-MLPA Kits ME001 and ME002 to analyze $\mathrm{CpG}$ island methylation in 34 tumor suppressor genes selected on the basis of their frequent epigenetic silencing in different tumors. These genes included CDKN2A, CDKN2B, BRCA1, BRCA2, MLH1 and PTEN. MS-MLPA probes generate 


\section{CDKN2A}

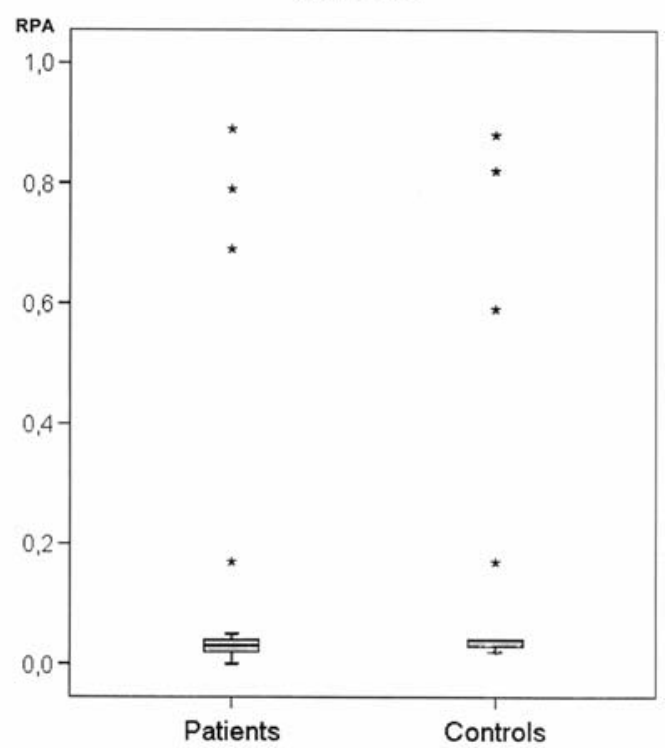

Figure 2. Promoter methylation levels are shown by the methylation-sensitive probe located in CDKN2A (MS-MLPA Kit ME001). The boxplot shows patients with malignant melanoma (left) and controls (healthy blood donors; right). The MLPA Kits contain probes specific to the target region of interest and reference probes located in chromosomal regions outside the target region. The calculated values (peak areas) for the target peaks are divided by the corresponding normalized quotient from all reference probes. The resulting quotient is called relative peak area (RPA; sequence pilot). The quotient RPA (patient)/RPA (control) was listed for each probe in the software, and showed no significant differences using MLPA Kits ME001 and ME002 (cut-offs were $\pm 20 \%$; not shown). Thus, the RPAs from patients were similar to the RPAs from the controls.

different signals depending on the methylation status of the site. Using this technique, we found no signals indicative of methylation in the promoter regions of the tumor suppressor genes, including CDKN2A, in either the patients or the controls (Fig. 2). Methylated control DNA (enzymatically-methylated human genomic DNA) was used as a positive control for methylation.

\section{Discussion}

Several genetic studies performed in our western Swedish population have revealed genetic variations specific to the region. In the case of a number of other hereditary diseases, such as psoriasis and hereditary breast and ovarian cancer, there is an increase in the incidence of founder mutations in the population $(10,11)$. Moreover, there is a higher incidence of malignant melanoma in western Sweden compared with other parts of the country. In addition, the incidence of socalled dysplastic nevi, which are regarded as a risk factor or even as a pre-stage of malignant melanoma, is more common in this region.

CDKN2A is the major melanoma predisposing gene and hereditary CDKN2A mutations have been demonstrated in a range of $25-40 \%$ in different populations. Despite large-scale efforts in the years since CDKN2A was discovered, no other high-penetrant susceptibility gene for hereditary melanomas has been identified (12). We previously reported a low frequency of mutations in the CDKN2A gene in our western Swedish population (1). Our aim was therefore to identify other mechanisms involved in the downregulation of the CDKN2A tumor suppressor gene. Consequently, we decided to investigate whether heritable epimutations are one of the causes of the silencing of the CDKN2A gene. In this study, we used two different technical approaches to detect changes in $\mathrm{CpG}$ methylation in the promoter region of the CDKN2A gene. Using these methods, we demonstrated a consistent lack of hypermethylation in patients with HMM in our western Swedish population. Germ-line methylation of the CDKN2A promoter is therefore not a common event in hereditary melanoma. This finding is in line with a recent study of germ-line promoter methylation in BRCA1-negative families with hereditary breast cancer. The authors found no evidence of epimutations of the BRCA1 promoter (13). The magnitude and precise significance of heritable epimutations in familial cancer therefore remains to be determined.

\section{Acknowledgements}

This work was supported by grants from the Swedish Cancer Society, the Assar Gabrielssons Foundation and The Health and Medical Care Committee of the Region Västra Götaland.

\section{References}

1. Erlandson A, Appelqvist F, Wennberg AM, Holm J and Enerbäck C: Novel CDKN2A mutations detected in western Swedish families with hereditary malignant melanoma. J Invest Dermatol 127: 1465-1467, 2007.

2. Hashemi J, Bendahl PO, Sandberg T, et al: Haplotype analysis and age estimation of the 113insR CDKN2A founder mutation in Swedish melanoma families. Genes Chromosomes Cancer 31: 107-116, 2001.

3. Chan TL, Yuen ST, Kong CK, et al: Heritable germline epimutation of $\mathrm{MSH} 2$ in a family with hereditary nonpolyposis colorectal cancer. Nat Genet 38: 1178-1183, 2006.

4. Hitchins MP, Wong JJ, Suthers G, Suter CM, Martin DI, Hawkins NJ and Ward RL: Inheritance of a cancer-associated MLH1 germ-line epimutation. N Engl J Med 356: 697-705, 2007.

5. Herman JG, Graff JR, Myöhänen S, Nelkin BD and Baylin SB: Methylation-specific PCR: a novel PCR assay for methylation status of CpG islands. Proc Natl Acad Sci USA 93: 9821-9826, 1996.

6. Esteller M, Tortola S, Toyota M, Capella G, Peinado MA, Baylin SB and Herman JG: Hypermethylation-associated inactivation of $\mathrm{p} 14(\mathrm{ARF})$ is independent of $\mathrm{p} 16$ (INK4a) methylation and p53 mutational status. Cancer Res 60: 129-133, 2000.

7. Hoque MO, Begum S, Topaloglu O, et al: Quantitation of promoter methylation of multiple genes in urine DNA and bladder cancer detection. J Natl Cancer Inst 98: 996-1004, 2006.

8. Nygren AO, Ameziane N, Duarte HM, et al: Methylation-specific MLPA (MS-MLPA): simultaneous detection of CpG methylation and copy number changes of up to 40 sequences. Nucleic Acids Res 33: e128, 2005.

9. Procter M, Chou LS, Tang W, Jama M and Mao R: Molecular diagnosis of Prader-Willi and Angelman syndromes by methylation-specific melting analysis and methylation-specific multiplex ligation-dependent probe amplification. Clin Chem 52: 1276-1283, 2006.

10. Enlund F, Samuelsson L, Enerbäck C, et al: Psoriasis susceptibility locus in chromosome region 3 q21 identified in patients from southwest Sweden. Eur J Hum Genet 7: 783-790, 1999.

11. Bergman A, Einbeigi Z, Olofsson U, et al: The western Swedish BRCA1 founder mutation 3171 ins 5 ; a 3.7 cM conserved haplotype of today is a reminiscence of a 1500-year-old mutation. Eur J Hum Genet 9: 787-793, 2001.

12. Bishop JN, Harland M, Randerson-Moor J and Bishop DT: Management of familial melanoma. Lancet Oncol 8: 46-54, 2007.

13. Chen Y, Toland AE, McLennan J, Fridlyand J, Crawford B, Costello JF and Ziegler JL: Lack of germ-line promoter methylation in BRCA1-negative families with familial breast cancer. Genet Test 10: 281-284, 2006. 